\title{
Design and Implementation of Rehabilitation Training and Positioning System Based on Multi-Sensor Information Fusion
}

\author{
Qiuzhan Zhou* \\ State Key Laboratory of Automotive Simulation and Control, Jilin University, Changchun 130022, China \\ College of Communication Engineering, Jilin University, Changchun 130022, China
}

Yongchao Xue \& Shuozhang Chen

College of Communication Engineering, Jilin University, Changchun 130022, China

Songling Zhang

The First Hospital of Jilin University, Changchun, 130021, China

\author{
Zongheng Lei \& Yujuan Si \\ College of Communication Engineering, Jilin University, Changchun 130022, China
}

ABSTRACT: Towards human motion intention recognition in active rehabilitation, an algorithm of pattern recognition and localization is proposed. Large changes in the overall action signal were paid more attention rather than accuracy of local signal. The ARM of Cortex-M3 core was used in data acquisition and the LabVIEW to program human-computer interaction interface. Meanwhile the technology of intelligent action decision based on Neural Networks Artificial (ANN) was used and the wavelet packet was used to extract the signal feature of actions. Combined with the rehabilitation staff attributes information such as age, gender, stride length of walk, run, down stairs and up stairs to get the count of the staff's actions and location. Experimental results show that the performance of this method is $100 \%$ tested by genetic parameters optimization and the accuracy rate is $96.6667 \%$ by ROC. The target can be located according to the track of the target. KEYWORD: Information Fusion; Rehabilitation training; ANN; location; wavelet package; LabVIEW

\section{INTRODUCTION}

In the field of medical training rehabilitation, it's a great reference for attending doctors and families to acquire information of patients timely and conveniently. For instance, the recovery data of patients with leg rehabilitation, the walk steps of everyday, the amount of jogging and the position indoors are significant data for the recovery of patients. On the one hand, most equipment without the function of indoor positioning are focus on the field of sports which also have a high price and cannot gather the information of people. As for the aspect of personnel positioning, there are mainly two categories of the indoor positioning: Local Positioning System (LPS) based on network technology and Pedestrian Navigation System based on inertial navigation technology (D.Gualda et al, 2014) (G.H. Tian et al, 2015). LPS is based on the base station with high costs which is not proper for daily use. In terms of PDR, it is complicated relatively and the accuracy of the sensors has a great impact on the calculation of the target's posture with accumulated deviation. The above tow methods, meanwhile, cannot recognize the different actions of people. On the other hand, most designs of rehabilitation training equipments are mechanical and passive with a high cost that are unable to collect the rehabilitation training data to be analyzed scientifically and effectively. Moreover, they are also without the function of indoor positioning ensuring the safety of patients. Considering the present situation, the paper puts forward a new method to design an innovative and low-cost rehabilitation training and positioning system, which is based on information fusion and pattern recognition.

\section{THE ACTION DATA ACQUISITION OF THE REHABILITATION}

\subsection{The Hardware of the Data Acquisition of Action Signals.}

The posture of gait, such as walk, jog, stand, upstairs, downstairs are recognized by the sensors of tri-axial accelerometer, tri-axial gyroscope and electronic compass (B. Ayrulu-Erdem et al, 2011). Each sensor would produce response signals with the patients' actions which are the key signals for us to extract the features of actions. The parameters of angular velocity, linear acceleration, the yaw of the north are measured by tri-axial gyroscope, tri-axial accelerometer and electronic compass respectively. The introduction of the sensors is as follows:

The model of tri-axial gyroscope, L3G4200D with the characteristics of low power consumption is produced by STM. It has a good performance in terms of sensitivity and stability and the data of gyroscope is used as auxiliary judgment. 
The tri-axial accelerometer, ADXL345 introduced by ADI with the output serial bus of I2C is developed based on the principle of MEMS which can identify the change of 0.25 degrees. As a result, it can detect the dynamic acceleration in fields of wearable and the signals of it are used as assistance to features extraction of action signals.

The chip HMC5883 launched by Honey Well with small size and low cost can achieve 1 to 2 degrees of the accuracy of $12 \mathrm{~A} / \mathrm{D}$.

\subsection{The Data Acquisition System Based on ARM.}

The microcontroller of STM32F103 with the core of Cortex-M3 was used as the MCU of the design and the power system includes $5 \mathrm{~V}$ and $3.3 \mathrm{~V}$. The entire system is a complete set of data acquisition, which uses the wireless serial port for data transmission.

Minimum system of single chip: To achieve the relevant functions, the ARM processor with the core of Cortex-M3 should be programmed. The series of STM32F1 belong to the 32 bit ARM microprocessor of low and middle -end product with the functions of USART, CAN, ADC, USB, I2C, timer, etc, which provide the access speed of $1.25 \mathrm{DMips} / \mathrm{MHz}$ and the function of DMA is helpful to the data acquisition in real time.

The system consists of the sensors and the processor is shown as fig.1:

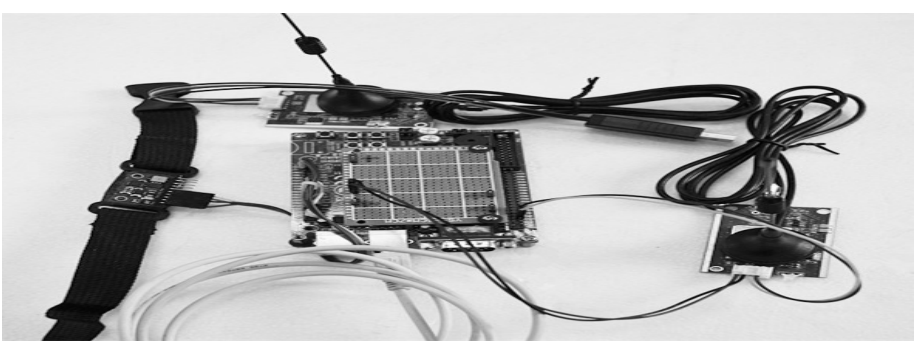

Fig.1 Lower computer hardware equipment

\subsection{The Design of Software of the Data Acquisition Model in Lower Computer.}

The overall program is used the structural of sequence under the development environment of KEIL. In order to give the initial value of the sensors, there are 5 seconds available for the MCU system and the sensors, meanwhile, processing 100 points collected for average. Then the signals acquired by the sensors are transmitted to the register of the serial port by DMA, which are sent to the host computer by wireless serial transceiver and at the same time, the lower computer continuous to acquire action signals. The process repeats all the times.

\subsection{The Display of the Software of Patients' Actions in Host Computer.}

The software in host computer is mainly programmed by LabVIEW, supplemented by MATLAB. The acquisition of action signals of the rehabilitation patients' is done by lower computer, which is transmitted to the upper computer to be processed by the software. The main functions of the software are visual design, signal display, data storage, etc. The whole interface of the software as shown in fig.2:

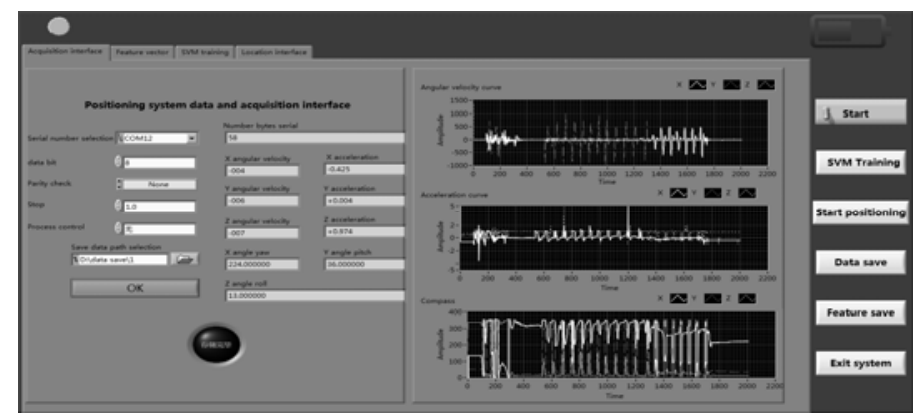

Fig.2 The recovery of the upper computer display

\section{THE CONSTRUCTION OF OVERALL SYSTEM}

The author has acquired the data of angular velocity, acceleration and the yaw of the north of $\mathrm{X}, \mathrm{Y}$ and $\mathrm{Z}$ axis in order to achieve the function of the changeable signals of the rehabilitation patients. The display part of the interaction interface programmed by LabVIEW can achieve the functions of action data acquisition, feature extraction, classification and recognition, positioning, etc.

The unit of data acquisition has play an important role between the sensors ahead and the software of the host computer, which is responsible for the configuration and control commands of the sensors, meanwhile, gathering the output signals of the sensors. And the functions of action count, the rehabilitation positioning algorithm, data acquisition and storage, interface display, feature extraction, etc. are completed by the software of the host computer. The software processes the data initially, then to generate the feature vectors that are sent to the BP network for training. Then we can accord the steps of the patient's and the changes of yaw to calculate the position of the rehabilitation. The functions all above are completed by the software on the host computer programmed by LabVIEW. The overall block diagram is shown in fig.3: 


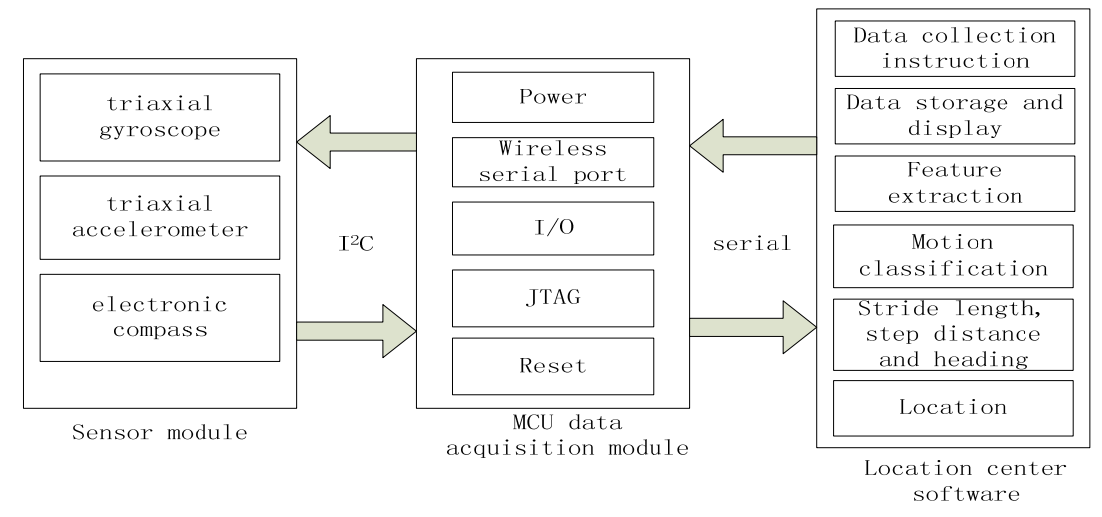

Fig.3 Overall working block diagram

\section{CLASSIFICATION OF ACTION RECOGNITION AND POSITIONING PRINCIPLE}

There are some information needed to be considerate including the indoor layout of the rehabilitation, gender, age, walking stride, running stride, up and down stairs forward distance, etc. before the start of the recognition and localization. In order to obtain the feature vector of different actions, the system of the rehabilitation training and location need to some methods to process the data gathered from the unit of data acquisition, including denoising, wavelet packet decomposition. We can recognize the classification of the patient's actions through sending the feature vectors of different actions to BP neural network, which can apply us the information for positioning. We can detect the gains through the following methods: the feature vectors of gait energy can be identified from the signal wave of the $\mathrm{X}$ axis angular velocity; the count of walk steps can be got from the signal wave of the $\mathrm{Y}$ axis accelerometer; as for turning, through the signal of $\mathrm{Z}$ axis of the electronic compass. All above methods are processed by the upper computer software. Then identify the actions of walking, jogging, downstairs and upstairs of the rehabilitation patients, which can be combined with the track of the rehabilitation and the indoor layer to locate the position of people. The specific algorithm is explained as follows.

\subsection{Action Recognition Based on the Theory of Wavelet Packet BP Neural Network.}

Wavelet packet analysis can be used to analyze the components of a specific frequency of signal, so it has a strong ability of localization analysis; BP neural network can be trained to minimize the error of the desired output vector and the actual output vector (M. Li et al, 2011). Different frequency bands of the signal of walking, jogging, downstairs and upstairs have different percentage. On the whole, these signals of actions are belong to low frequency signal and most of the signal is concentrated in the range of
$0 \mathrm{~Hz}$ to $25 \mathrm{~Hz}$. The author has employed a lot of experiments and the results show that the feature vectors of actions can be well recognized through wavelet packet decomposes the coefficients before extracting the angular velocity of $\mathrm{X}$ axis, then keep the useful wavelet coefficients and delete the useless wavelet coefficients as noise. After the procedure, we need to reconstruct the new signals. In this paper, we use the following method to extract the wavelet coefficients (H.F. Xing et al, 2007) (Y. Mallet et al, 1997) (N. Lovell et al, 2007).

Use wavelet packet to extract the feature of the signals of patient's actions including signals of walking, jogging, upstairs and downstairs. The steps are as follows:

(1) The signals of actions are acquired and processed by the hardware system of the lower processor and the front-end signal of the target is represented by $\mathrm{S}$. We use $d b 06$ as the base function to decompose the original signals in three layer wavelet packet transform. The $(i, j)$ is used to represent the $i$ layer and $j$ node $(i=0,1,2, \ldots, N$, $\left.j=0,1,2, \ldots, 2^{N}-1\right)$. The symbol $X_{i, j}$ represents the coefficients of wavelet decomposition of layer $i$, band $j$.The specific form of decomposition is shown in fig. 4:

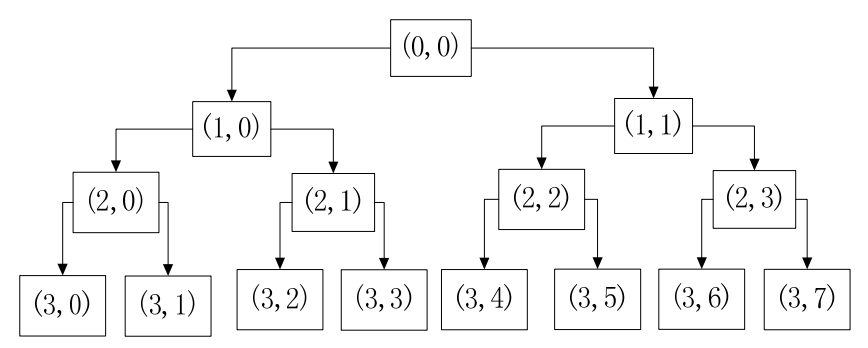

Fig.4 Wavelet packet coefficient decomposition

(2) Reconstruct the coefficients $X_{i, j}$ of last layer and the new formula of reconstructed signal as follows: 


$$
S=S_{i, 0}+S_{i, 1}+\ldots+S_{i,\left(2^{N}-1\right)}
$$

The energy of $S_{i j}$ is represented by $E_{i j}$ and the formula of $E$ is shown as follow:

$$
E_{i, j}=\int\left|S_{i, j}(t)\right|^{2} d t=\sum_{k=1}^{n}\left|x_{j, k}\right|^{2}
$$

In the formula, the symbol $x_{j, k}\left(j=0,1,2, \ldots, 2^{N}-1, k=1,2,3, \ldots, n\right)$ represents the component amplitude of $S_{i j}$.

(3) The energy $E_{i j}$ calculated by the above formula is seen as feature vector and we can draw the formula below:

$$
T=\left[E_{i, 0}, E_{i, 1}, \ldots, E_{i,\left(2^{N}-1\right)}\right]
$$

The above feature vectors are normalized and due to the power spectrum of the jogging are obviously higher than that of walking, the power spectrum varies a great deal from actions to actions. So we need to normalize the feature vectors in order to reduce the error of BP neural network. At the same, the speed of training increase to some extent with a good generalization. $T$ 'is the normalized vector.

$$
\begin{aligned}
E & =\left(\sum_{j=0}^{2^{N}-1}\left|E_{i j}\right|^{2}\right)^{\frac{1}{2}} \\
T^{\prime} & =T / E=\left[E_{i 0} / E, E_{i 1} / E, \ldots, E_{i\left(2^{N}-1\right)} / E\right]
\end{aligned}
$$

\subsection{Action Recognition Based on BP Neural Network.}

As for the classification of the rehabilitation, we combine the pattern recognition technology with the feature vectors calculated by above formula to process. The small training set would appear considering the time-effectiveness according to the matter of fact. The target recognition algorithm based on BP neural network that we choose is composed of construction, network training and network classification. The feature vector is used as the input of BP neural network (M.Song, 2005) (R.W. Nie, 2007) (X.L. Tao, 2007).

(1) The construction of BP neural network

The design of input network layer: the author use the feature vectors that are extracted from original signals and the number of input are $2^{N}$ ( $N$ represents the total layer of wavelet packet).

The design of output network: the action of stand needn't be classified by BP network apart from the action of walking, jogging, upstairs and downstairs.
Because there are only some drift signals of the output when the patients stand and theses signals can be directly solved by the threshold value in the software. According to the experiment data, the number of four kinds of actions vectors we draw are 3, 4, 4, 3 and get the specific values of the support vector are obtained.

The design of hidden network layer: The number of nodes in the hidden network layer is determined by the empirical formula $l=\sqrt{m+n}+\alpha$ and $\mathrm{n}$, $\mathrm{m}$ represents the number of input and output layers respectively (M.Song, 2005).

(2) The data training of BP network

We do the following experiments to acquire the training data of BP network in order to establish the model of classification. Before the experiment, we need to choose a certain place, orientation as well as testing personnel in advance. Each participant was asked for 5 times. The experiment was employed as follows.

Step1: walk 32 steps from the south to the north with left foot first.

Step2: jog 31 steps from the north to the south with left foot first.

Step3: upstairs from the east to the west on 11 flights of stairs with left foot first.

Step4: downstairs from the west to the east on 11 flights of stairs with left foot first.

Step5: repeat step1 to step4.

And the data above is saved as the attribute vector and label vector.

The initial value of network weight is satisfied by the random number in $(-1,1)$ (R.W. Nie, 2007) (X.L. Tao, 2007), which can guarantee the speed and accuracy of the network training. Besides, through the small sample training data can increase the speed of training. The matrix of attribute vector and label vector is sent to the MATLAB training module of $\mathrm{BP}$ network to complete the data training.

(3) The classification of BP network

After training, the BP neural network can meet the requirements of target recognition and the feature vector extracted from the original data can be directly sent to BP network to complete the recognition of the target. Then the classifier will output the action category automatically, which lays a foundation for the positioning.

\subsection{The Determination of Direction and Turning.}

Judging the turning of the rehabilitation staff: The method of "power spectrum vector time chasing" was applied to distinguish the turning of the rehabilitation staff in our design. As for the data of original angle, $X_{t}=\left\{X_{t 1}, X_{t 2}, X_{t 3}, \ldots, X_{t n}\right\}$, we have the following described formulas : 


$$
\begin{gathered}
\overline{X_{t_{1}}}=\left\{\overline{x_{t_{1}} 1}, \overline{x_{t_{1}}}, \ldots, \overline{x_{t_{1} m}}\right\} \\
\overline{X_{t_{2}}}=\left\{\overline{x_{t_{2} 1}}, \overline{x_{t_{2}}}, \ldots, \overline{x_{t_{2} m}}\right\}
\end{gathered}
$$

In the above formulas, 30 times and 10 times of the average power spectrum vector were represented by $\overline{X_{t_{1}}}$ and $\overline{X_{t_{2}}}$ respectively. For $t_{1}-t_{2}>0$, we set:

$X_{\Delta t}=\bar{X}_{t_{2}}-\bar{X}_{t_{1}}=\left\{x_{\Delta t 1}, x_{\Delta t 2}, x_{\Delta t 3}, \ldots, x_{\Delta t m}\right\}$

Then, another function was structured as follows.

$y_{\Delta t i}=\left\{\begin{array}{l}1,\left|x_{\Delta t i}\right| \geq x_{\text {threshold }}, \\ 0,\left|x_{\Delta t i}\right|<x_{\text {threshold }},\end{array} i=1,2,3 \ldots m\right.$

When the action of turning happens, the value $y_{\Delta t_{i}}$ would exceed the corresponding threshold, which can be captured by experiment.

Judging the direction of turning of the rehabilitation staff: In short, the direction of turning can be judged through the changes of compass. The angle of the compass is absolute for the north, and after wearing with initialization, the angle is zeroed by software in host computer, which was seen as the default direction in zero. When turning the left or right, the electronic compass would show a positive or a negative change, which is illustrated in Fig.5.The figure shows that the participant wearing the device is in position A, initialized to zero at the moment. The absolute angle to the north decreases with the staff turns left, and the angle of the moment minus the angle of $\mathrm{A}$ is a negative value, whereas turning right similarly.

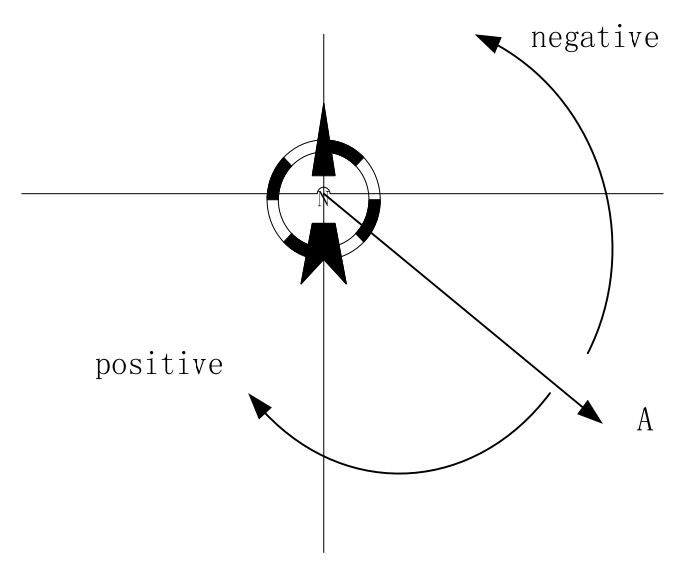

Fig.5 Decision of turning direction

\section{SIMULATION OF REHABILITATION PERSONNEL}

\subsection{Simulation of Walking Signals of Rehabilitation Personnel.}

The signals of tri-axial angular velocity meter, accelerometer and electronic compass will change due to the actions of staff. The following experiment was carried out in our design. The participant worn the device on the heel of his right foot and walked 32 steps from the south to the north with left foot first. The signals of the actions were transmitted to the experimenter's notebook computer by using the wireless serial port which were captured and displayed by the software in upper computer. The signal sampling rate is set at $50 \mathrm{~Hz}$ and the initial waveform is extracted from 20 seconds to 80 seconds is shown in Fig. 6.

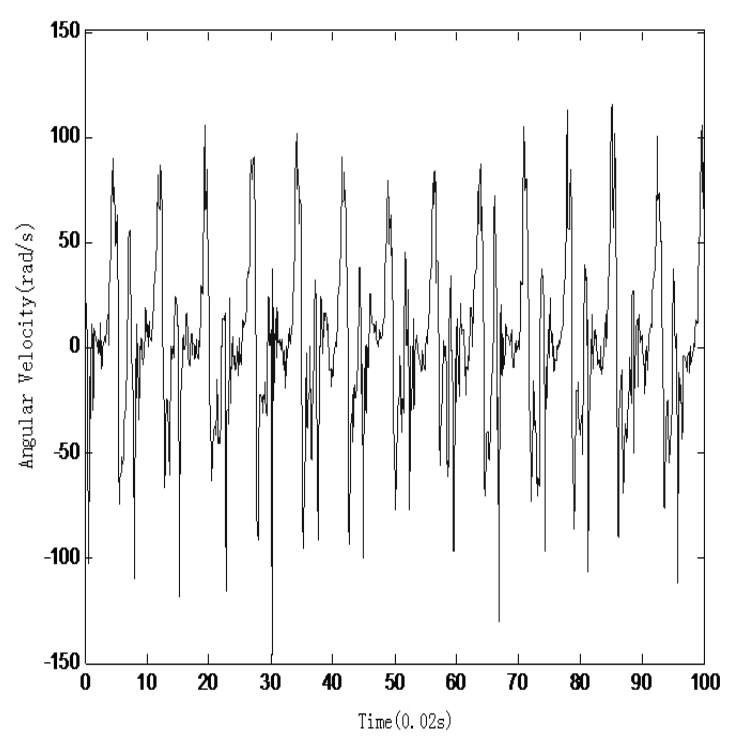

Fig.(a) $\mathrm{X}$ axis angular velocity

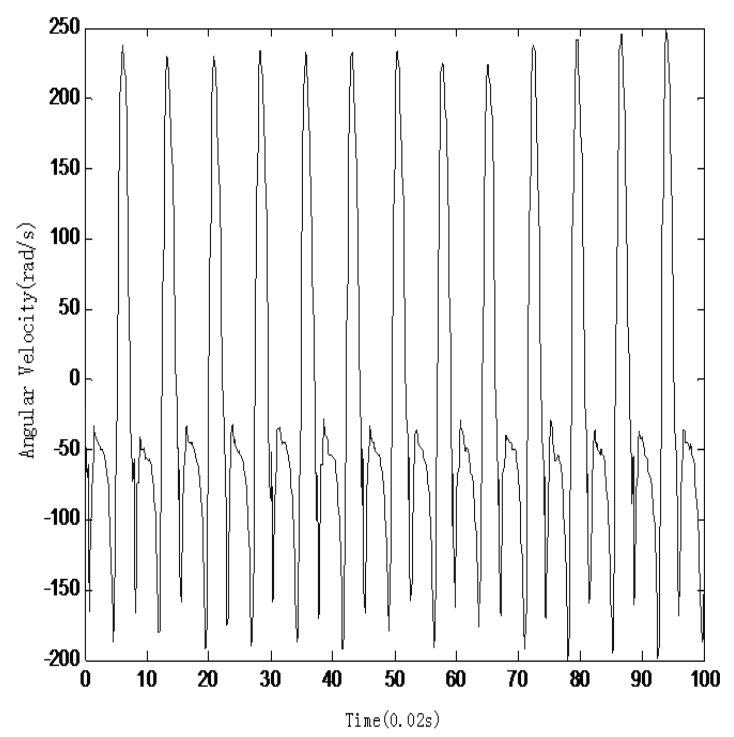

Fig.(b)Y axis angular velocity 


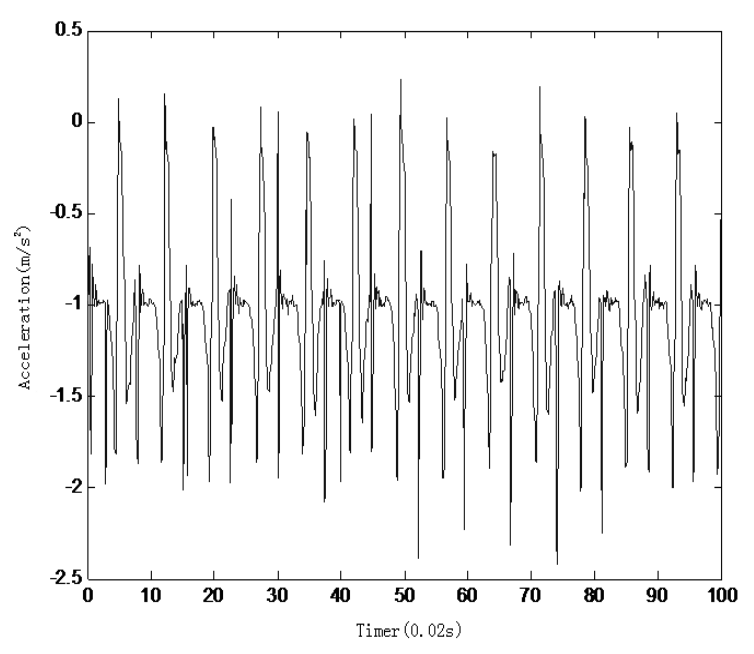

Fig.(c) $\mathrm{X}$ axis acceleration

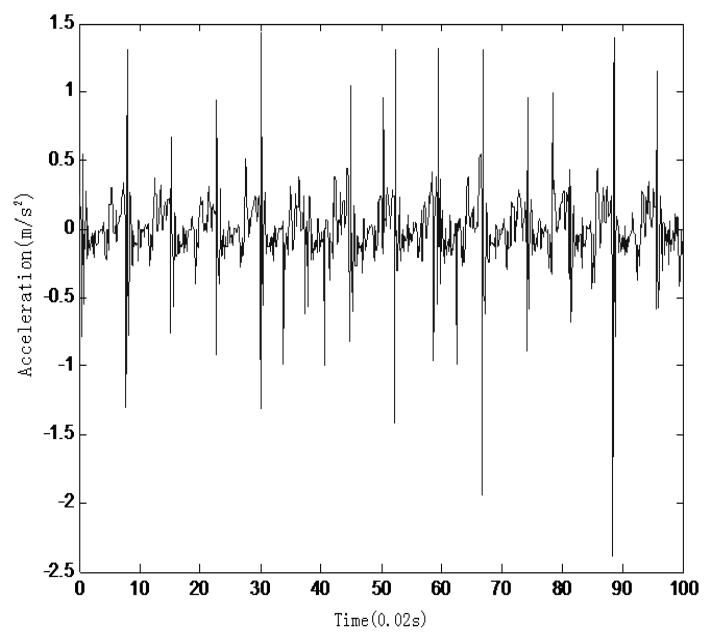

Fig.(d) $\mathrm{Y}$ axis acceleration

Fig.6 The original signal of walk in $20 \sim 80$ s

Firstly, we can get the information that there is an obvious variation trend of the signals of each sensor in the $X$ axis (A.M. Sabatini et al, 2005), and the signals are in the low frequency with a state of nonstationary. There are apparent signals of acceleration and angular velocity in $\mathrm{X}$ axis at the moment of foot landing on the ground (F.E. Zajac et al, 2003). Besides, the frequency of walking is lower than that of jogging. Secondly, in general, the signals are similar to those of $\mathrm{X}$ axis when compared with $\mathrm{Y}$ axis, but the angular velocity and acceleration of the waveform also show other regularity. Observed from the macro, when patients walk normally, it will exhibit a shift inclined away from the direction of the footsteps which has the characteristics of stability and uniqueness. In our experiment, the shifts are represented by $\mathrm{Y}$ axis and the peaks of $\mathrm{Y}$ axis of accelerometer are equal to the numbers of steps of the staff exactly according to large numbers of experiments and statistics. As for signals of $\mathrm{Z}$ axis, it suggests a stable and periodicity tendency which can be used to determine the direction by the changes of angle.

Through the above analysis, we can use the following method to detect gait on the basis of a periodic signal: firstly, obtain feature vector through $\mathrm{X}$ axis angular velocity signal and then get gaits through $\mathrm{Y}$ axis accelerometer signal, finally acquire direction information in line of $\mathrm{Z}$ axis signal.

\subsection{Analysis of Walking Signals and the Extraction of Gaits.}

We should filter the signals because of lots of noise components (Hausdorff J.M et al, 2001); otherwise, it will cause adverse effects on the feature extraction of signal. According to a large number of experimental data and documents, the male's walking frequency is $0.91 \sim 1.03 \mathrm{~Hz}$ and the female is $0.98 \sim$ $1.10 \mathrm{~Hz}$ (L. Frank et al, 2007).Consequently, we design a Bessel band pass filter with high pass cutoff $25 \mathrm{~Hz}$ (B. Auvinet et al, 2002) and low pass $0.5 \mathrm{~Hz}$ of 5 -order which used to the signal of $\mathrm{X}$ axis angular velocity.

For the number of steps, we take the algorithm of wavelet to extract the peaks of signals of $\mathrm{Y}$ axis of accelerometer filtered by the wave as is shown in figure 7 :

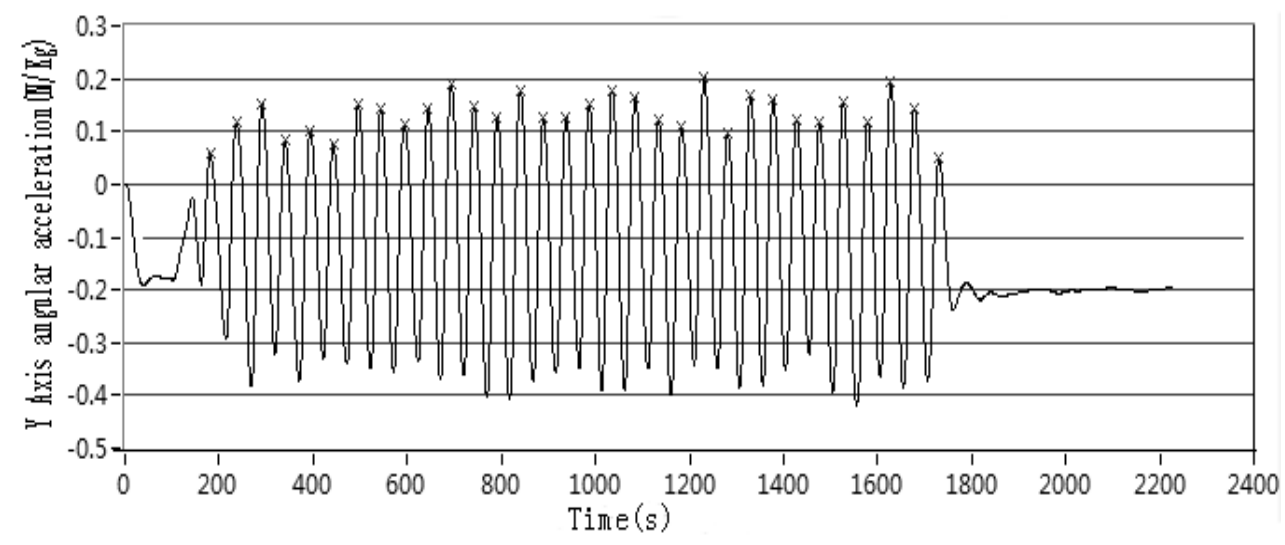

Fig.7 Extraction peaks of $\mathrm{Y}$ axis accelerometer 
The numbers of the peaks extracted from the filtered signal are equal to the steps of our staff in the experiment. In conclusion, we can use the signal of $\mathrm{Y}$ axis of accelerometer to conclude the staff's gaits.

\section{ANALYSIS OF THE CHARACTERISTIC ENERGY OF SIMULATED THE REHABILITATION}

Select the data between different actions to extract the feature of different sections of the data stored in each segment. Because of the noise, use low pass filter designed previously for noise reduction with the parameters of sampling rate 200, low cutoff frequency $10 \mathrm{~Hz}$ and the 5-orderand then process the data of electronic compass. Keep staff still and extract 30 numbers of the data of the electronic compass in order to calculate its average value, which subtract the data of compass to get the relative angle value to get the direction information.

Tab.1 Personnel action characteristic energy distribution

\begin{tabular}{|c|c|c|c|c|c|c|c|c|}
\hline \multicolumn{2}{|c|}{ Action state } & Band 1 & Band 2 & Band 3 & Band 4 & Band 5 & Band 6 & Band 7 \\
\hline \multirow{5}{*}{ walking } & Action1 & 0.44785 & 0.83951 & 0.06655 & 0.29251 & 0.01237 & 0.05504 & 0.03853 \\
\hline & Action2 & 0.31915 & 0.85005 & 0.05022 & 0.23177 & 0.04348 & 0.05476 & 0.03071 \\
\hline & Action3 & 0.44087 & 0.95321 & 0.06553 & 0.16003 & 0.01669 & 0.04913 & 0.02794 \\
\hline & Action4 & 0.37971 & 0.91372 & 0.06184 & 0.12185 & 0.01082 & 0.041 & 0.02155 \\
\hline & Action5 & 0.46101 & 0.94808 & 0.0682 & 0.25347 & 0.01415 & 0.06514 & 0.04334 \\
\hline \multirow{5}{*}{ running } & Action1 & 0.38725 & 0.80015 & 0.27822 & 0.3657 & 0.13568 & 0.17084 & 0.29232 \\
\hline & Action2 & 0.25333 & 0.74846 & 0.25163 & 0.50174 & 0.15103 & 0.13297 & 0.28078 \\
\hline & Action3 & 0.33726 & 0.75631 & 0.22856 & 0.39166 & 0.12604 & 0.22284 & 0.20754 \\
\hline & Action4 & 0.31763 & 0.76662 & 0.27147 & 0.50322 & 0.20114 & 0.22508 & 0.33874 \\
\hline & Action5 & 0.40262 & 0.64527 & 0.20061 & 0.26708 & 0.24725 & 0.33887 & 0.36604 \\
\hline \multirow{5}{*}{ upstairs } & Action1 & 0.6934 & 0.64762 & 0.06357 & 0.02016 & 0.00512 & 0.02136 & 0.12006 \\
\hline & Action2 & 0.77979 & 0.61529 & 0.06408 & 0.05184 & 0.01999 & 0.0588 & 0.15198 \\
\hline & Action3 & 0.55547 & 0.65883 & 0.08512 & 0.07832 & 0.06103 & 0.0712 & 0.1089 \\
\hline & Action4 & 0.6862 & 0.64282 & 0.07948 & 0.10852 & 0.0601 & 0.07135 & 0.13164 \\
\hline & Action5 & 0.69376 & 0.77433 & 0.08479 & 0.01733 & 0.07515 & 0.08573 & 0.22403 \\
\hline \multirow{5}{*}{ downstairs } & Action1 & 0.47169 & 0.70768 & 0.19367 & 0.37819 & 0.02699 & 0.11857 & 0.16163 \\
\hline & Action2 & 0.43136 & 0.74404 & 0.28365 & 0.35052 & 0.05045 & 0.15988 & 0.17005 \\
\hline & Action3 & 0.45381 & 0.79083 & 0.19644 & 0.32474 & 0.01758 & 0.13895 & 0.17062 \\
\hline & Action4 & 0.42553 & 0.63518 & 0.2296 & 0.41201 & 0.13509 & 0.10259 & 0.16515 \\
\hline & Action5 & 0.43463 & 0.7489 & 0.22667 & 0.39416 & 0.03849 & 0.16512 & 0.1215 \\
\hline
\end{tabular}

We use the VI based on the theory of wavelet transform of the wavelet toolbox in using LabVIEW which can restore the continuous waveform via time signal synthesis function. Then use "Wa Denoise.VI" to reduce the noise whose theory has been introduced above. In this procedure, select the appropriate parameters to achieve the best results, and then put the data into the feature extraction function. Take the example of the angular velocity $\mathrm{X}$ axis described as follows:

Using 3 layers of wavelet packets to decompose, the $\mathrm{X}$ axis of a single action of a gyroscope signal was divided into several segments which were extracted to energy characteristics respectively. In order to reduce resources occupied by LabVIEW and promote the speed of process, we use the method of hybrid computing by MATLAB and LabVIEW which calls the "wpdec" function of wavelet packet tool box in MATLAB by LabVIEW to extract the coefficients of each layer, improve the interface to call the sub VI. As a result, we complete the feature extraction of $\mathrm{X}$ axis angular velocity.

In this paper, we will analyze the energy of the standing, walking, running, upstairs and downstairs.
In the case of standing, the BP network turns out to be no solution and enters a static state. Other four cases can be analyzed from the aspect of energy. After the signal is extracted and normalized by the filtering feature, 5 action feature vectors are given, as shown in table 1 after a series of process such as extracting, filtering and normalizing.

We can draw from the table that the energy distribution of the same movement shows a great similarity, and different personnel also have similar distribution of energy. But there are certain differences between different movements. Select two kinds of action feature to analyze and others are similar. As shown in Figure 8, when the staff begins to run, the energy shifts to the high frequency components with the increase of high frequency decomposed by wavelet packet and the energy increasesinband3, 5 and 7. When upstairs, as shown in Figure 9, the low frequency increased compared to walking and the distribution of energy in band 1, 2 is equal respectively. 


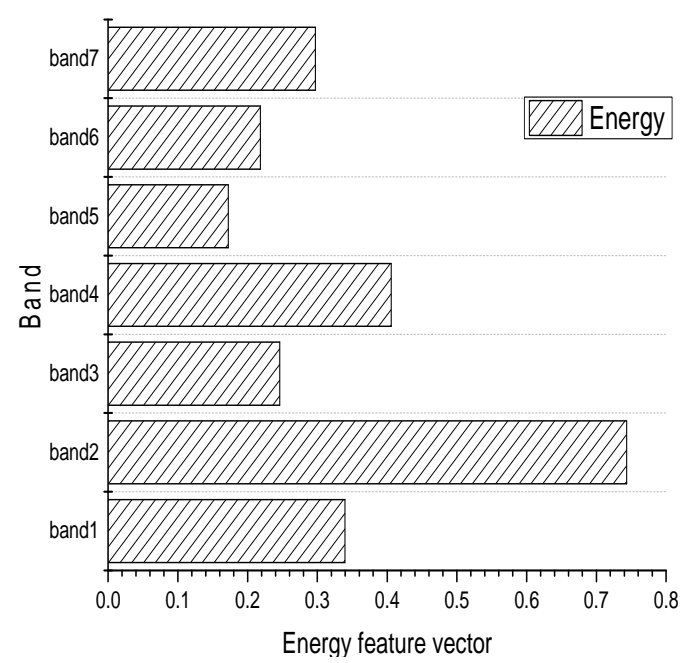

Fig.8 Running state energy distribution

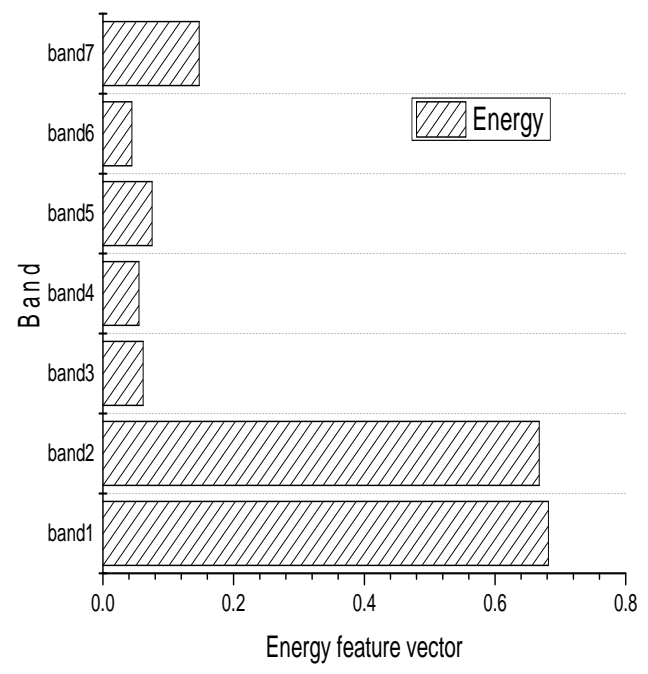

Fig.9 Upstairs state energy distribution

\section{ANALYSIS OF CLASSIFICATION OF BP NETWORK}

In order to evaluate the acceptability of classification, the results of the BP network classification were tested and evaluated by the authors. Select a sort of test data and reduce the penalty factor as small as possible to enhance the generalization of small sample. Through the algorithm of genetic parameters to optimize the parameters and get the optimal parameters as follows of Figure 10: through the experiment, the author got 14 vectors of walking, 12 vectors of running, 10 vector upstairs and 15 vectors downstairs. The accuracy of classification is $100 \%$.

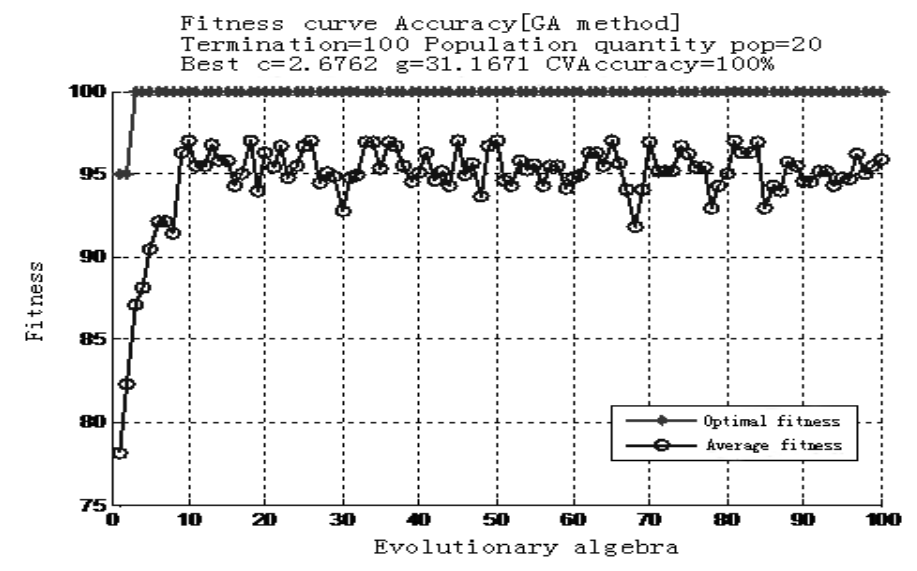

Fig.10 Genetic algorithm BP network parameters optimization

To further verify the accuracy of classification, the author used another set of experimental data of walking and running to evaluate the validation and other action signals were processed similarly. The ROC curve is based on a series of different binary mode (boundary value or decision threshold) (Y.S. Wang et al, 2007) and the vertical axis represents the real rate and the horizontal axis represents the false positive rate. The curve is closer to the upper left corner, which indicates that the effect of classification is better. Use the ROC curve as the evaluation of the results of the classification (B.K. Wang et al, 2006), which can better explain the result. The author selected the ROC curve diagrams of walking and running as shown below 11 .

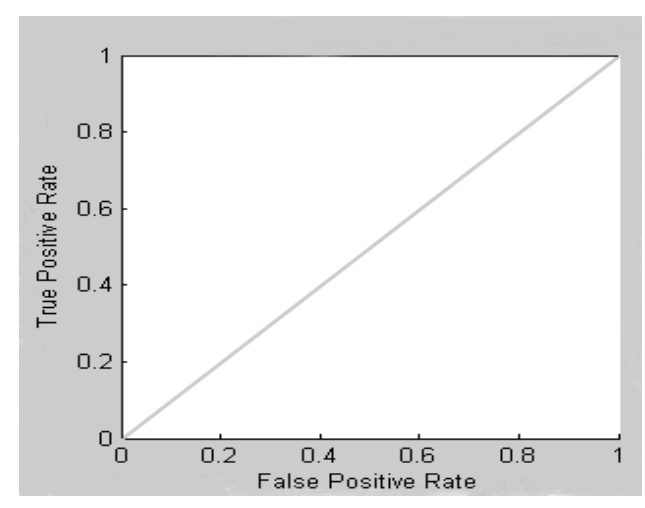

Fig.(a) ROC of walking

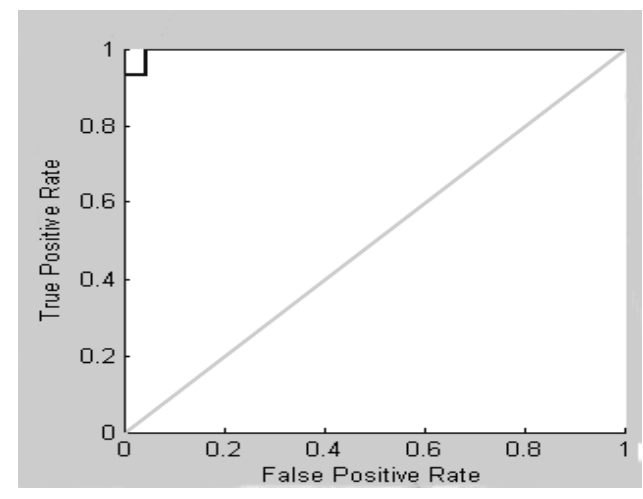

Fig.(b) ROC of running 
Fig.11 Classification Results of ROC curve

After the author got a classification model using the training data, the results of the classification were evaluated by the ROC and the accuracy rate of the result is $96.6667 \%$ (58/60) (classification). From the above curves can be drawn, an error classification of the action of running occurred and the results of the whole classification are more ideal.

\section{THE INTERFACE OF SIMULATED REHABILITATION PERSONNEL POSITIONING}

The location of the staff can be used by the information obtained by the above analysis and the vectors of the positions were stored in the array of the program. The main input parameters are test personnel's properties as shown in fig.12. The table 2 lists the 11 kinds of action states that are represented by symbols and numbers:

Tab.2 Numerical implication

\begin{tabular}{llll}
\hline Meaning & Number & Meaning & Number \\
\hline Walking & +1 & Walking & -1 \\
forward & backward & Turn right & -2 \\
Turn left & +2 & Run backward & -3 \\
Run for- & +3 & Run right & -4 \\
ward & +4 & Downstairs & -5 \\
Run left & +5 & & \\
Upstairs & +5 & & \\
Stand & 0 & & \\
\hline
\end{tabular}

The tab of location and counting is shown as follows and the program can drew the track of the staff through the detection of the left or right of the staff, which can be seen as the simulated trajectory of the staff. The program should be entered the properties of the staff and the block indicates the location of the staff. The array shows the previous status of the staff and the LEDs on the panel display personnel current state. The interface of the location is shown in figure 12:

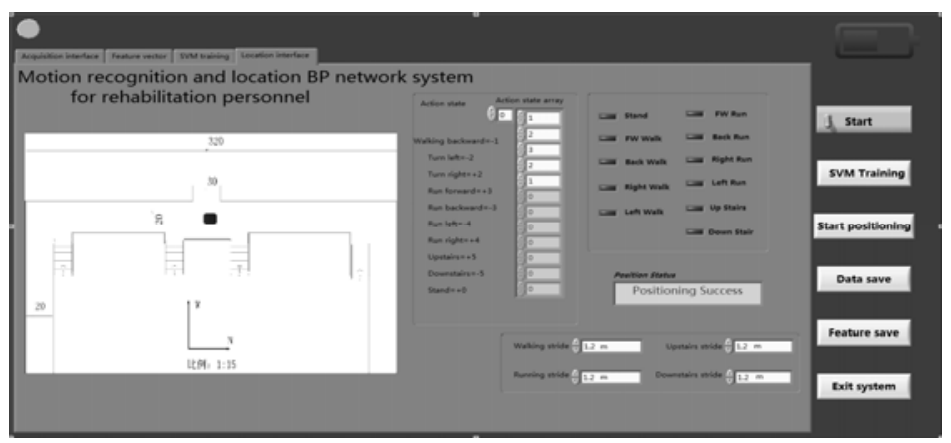

\section{SUMMARY}

Based on multi sensors information fusion, the author studied and designed the training location system of rehabilitation which is different from the traditional motion recognition and positioning principle and the signal analysis takes advantage of the overall signal rather than the details of the signal itself. So the method overcomes the impact of the sensor drift of traditional techniques that focus on the accuracy signal itself. Firstly, use the multi sensor data acquisition to acquire different action signals with the Butterworth filtering processing, and then DB6 wavelet packet decomposition and reconstruction is used to extract feature vectors. Finally recognize the action and then locate the staff indoor base on the BP network. The accuracy of recognition is as high as $96.6667 \%$ and the design can describe the simulated trajectory of the staff indoor.

\section{ACKNOWLEDGEMENTS}

The paper was funded by the Special Major Instrument of Jilin Province (20150203018GX), China Postdoctoral Science Foundation Project (2015m570272) and Provincial industry innovation special funds in Jilin Province (2016C046-1). Thanks for the co-authors of this paper. Zhou Qiuzhan (1974- ), correspondence author, male, associate professor, the main research direction: weak signal detection. E-mail: zhouqz@jlu.edu.cn.

\section{REFERENCES}

A.M. Sabatini, et al. Assessment of walking features from foot inertial sensing, J. Biomedical Engineering, IEEE Transactions on,. 52 (2005) 486-494.

B. Auvinet, et al. Reference data for normal subjects obtained with an accelerometric device, J. Gait \& Posture. 16 (2002) 124-134.

B. Ayrulu-Erdem, B. Barshan, Leg motion classification with artificial neural networks using wavelet-based features of gyroscope signals, J. Sensors. 2(2011): 1721-1743.

B.K. Wang, Z.J. Xue, etal.Application of ROC curve to optimizepattern classification algorithm, J. Progress in Natural Science Materials International. 11 (2006) 1511-1516.

D.Gualda, et al. Locally-referenced ultrasonic-LPS for localization and navigation, J. Sensors. 11(2014) 21750-21769.

F.E. Zajac, R.R. Neptune, S.A. Kautz, Biomechanics and muscle coordination of human walking, J. Gait \& Posture. 17 (2003) 1-17.

G.H. Tian, Q.D. Zhang, Research on integrated positioning of PDR and GPS based WT-UKF, J.Control and Decision. 01(2015) 86-90.

Hausdorff J.M., et al., When human walking becomes random walking: fractal analysis and modeling of gait rhythm fluctuations, J. Physica A: Statistical mechanics and its applications. 302 (2001) 138-147.

Fig.12 Location interface 
H.F. Xing, F. Li, Y.L. Liu, Wavelet denoising and feature extraction of seismic signal for footstep detection, C. Wavelet Analysis andPattern Recognition,2007. ICWAPR'07. International Conference. 2007.

L. Frank, et al., Urban form relationships with walk trip frequency and distance among youth, J. American journal of health promotion. 21 (2007) 305-311.

M. Li, C.L. Jiang, et al. Ground Moving Target Identification Based on Neural Network , J. IEEE.18 (2011) 439-442.

M.Song, Research on target recognition technology based on Neural Network, D.Nanjing: Nanjing University of Science and Technology. 2005.

N. Lovell, et al. Accelerometry based classification of walking patterns using time-frequency analysis, C. Engineering in Medicine and Biology Society, 2007. EMBS 2007. 29th Annual International Conference of the IEEE. 2007.

R.W. Nie, Detecting and controlling network technology with multisensor system, D.Nanjing:Nanjing University of Science and Technology. 2007.

ST, 018750, DevicesA.L3G4200_AN3393, Datasheet, S. Italy:ST, 2010.

ST, 14611, DevicesA.STM32F10Xreference manual_Rev11, Datasheet, S. Italy: ST, 2015.

X.L. Tao, Research and implementation of target recognition and personnel positioning algorithm based on ground motion, D.Nanjing: Nanjing University of Science and Technology,2007.

Y. Mallet, et al. Classification using adaptive wavelets for feature extraction, J. Pattern Analysis and Machine Intelligence, IEEE Transactionson. 10(1997) 1058-1066.

Y.S. Wang, B.Y. Xie,et al.Application of ROC curve analysis in evaluating the performance of alien species' potential distribution models, J.BIODIVERSITY SCIENCE. 04 (2007) 365-372. 